\title{
Hypoadiponectinemia: Association with risk of varying degrees of gestational hyperglycemia and with maternal ethnicity
}

\author{
Xinhua Chen ${ }^{1 *}$, Theresa O. Scholl ${ }^{1}$, T. Peter Stein ${ }^{2}$ \\ ${ }^{1}$ Department of Obstetrics and Gynecology, School of Osteopathic Medicine, University of Medicine and Dentistry of New Jersey, \\ Stratford, USA; *Corresponding Author: chenx1@umdnj.edu \\ ${ }^{2}$ Department of Surgery, School of Osteopathic Medicine, University of Medicine and Dentistry of New Jersey, Stratford, USA
}

Received 3 February 2012; revised 10 March 2012; accepted 13 April 2012

\section{ABSTRACT}

Objective: The aims of this study were to examine the influence of maternal hypoadiponectinemia on the risk of varying degrees of gestational hyperglycemia as well as on ethnic differences in circulating levels of adiponectin. Methods: A case-control study nested within a prospective cohort of healthy pregnant women compared those who developed gestational diabetes mellitus (GDM, $N=80$ ), or had an impaired glucose challenge test (GCT) non-GDM (defined as $1 \mathrm{hr}$ plasma glucose $>140 \mathrm{mg} / \mathrm{dl}$ after a 50-g oral glucose load when screening for GDM but did not meet the diagnostic criteria for GDM by a diagnostic oral glucose tolerance test, $N=128)$ to normal controls $(N=557)$. Concentrations of serum adiponectin were determined at entry $\left(\sim 17\right.$ weeks) and during the $3^{\text {rd }}$ trimester. Results: With multivariable adjustment, entry hypoadiponectinemia (the lowest tertile vs. other tertiles pooled) was strongly associated with an increased risk of GDM (AOR 2.68, 95\% Cl 1.62 - 4.41) and with impaired GCT non-GDM (AOR 1.58, 95\% Cl 1.05 - 2.38). This association persisted after adjustment for pregravid BMI despite the fact that the risk in obese women with low adiponectin was higher. Similar data were obtained during the $3^{\text {rd }}$ trimester. The concentration of adiponectin during the $3^{\text {rd }}$ trimester was significantly lower in African Americans compared to Hispanics or Caucasians and this difference was detectable in both cases and controls ( $p<0.05$ or $p<0.01$ for each). Conclusions: The present results identified a unique graded association between hypoadiponectinemia during early pregnancy with risk of GDM and less severe hyperglycemia non-GDM inde- pendent of maternal BMI. The relationship persisted to the $3^{\text {rd }}$ trimester. African American women have lower adiponectin level than Caucasian or Hispanic women. Hypoadiponectinemia may play a significant pathophysiological role in the development of GDM and of less severe gestational hyperglycemia.

Keywords: Gestational Diabetes Mellitus; Impaired Glucose Challenge Test; Ethnic Difference;

Hypoadiponectinemia

\section{INTRODUCTION}

Adiponectin is an important adipokine specially secreted by adipocytes $[1,2]$. It exhibits potent anti-inflammatory and atheroprotective effects in vascular tissue and is a regulator of insulin sensitivity and glucose homeostasis [3-5]. Low concentrations of circulating adiponectin in mid to late pregnancy are consistently associated with increased risk of gestational diabetes mellitus (GDM) [6-9]. Whether the association of hypoadiponectinemia with risk of GDM is independent of maternal adiposity is uncertain [6-9]. It is also not known if hypoadiponectinemia is associated with the risk of developing the less severe form of gestational hyperglycemia. Glucose intolerance that is less severe than overt GDM has been linked with an increased risk of adverse pregnancy outcomes as well as with abnormalities in glucose and fat metabolism [10-12].

There are racial/ethnic differences in the body composition, insulin resistance, the prevalence of metabolic syndrome and other obesity related chronic diseases [13-14]; the underlying mechanism(s) are not fully understood. Adiponectin is a useful marker because a reduced concentration is strongly associated with the risk of type 2 diabetes, hypertension and other cardiovascular conditions $[3,15]$, whereas increased levels are related to 
a reduction in diabetes risk [16]. Whether there are ethnic differences in maternal serum adiponectin level during either early or late pregnancy has not been studied. Thus, our primary aim was to investigate whether maternal hypoadiponectinemia during early gestation was not only associated with risk of GDM but also with less severe hyperglycemia, and if this association is independent of maternal BMI. The secondary aim was to determine whether there is a detectable difference in maternal serum adiponectin concentration among ethnic minority groups

\section{MATERIALS AND METHODS}

We conducted a case-control study nested within the Camden Study, a prospective cohort study of pregnancy outcome and complications in young, generally healthy women residing in one of the poorest cities in the continental United States [17]. The institutional review board at the University of Medicine and Dentistry of New Jersey approved the study protocol. Informed written consent was obtained from each participant after explanation of the nature and purpose of the study.

Data of socioeconomic, demographic and lifestyle were obtained by interview at entry to care (14.2 \pm 0.19 week of gestation, mean \pm SE), and updated at weeks 20 and 28 gestation. Ethnicity was self-defined. BMI was computed based on self reported pregravid weight and measured height at entry to prenatal care $\left(\mathrm{kg} / \mathrm{m}^{2}\right)$. Maternal obesity was defined as BMI $\geq 30$. Blood samples collected at entry to care $(17.1 \pm 0.19$ week) and during the $3^{\text {rd }}$ trimester $(30.41 \pm 0.12$ weeks, mean $\pm \mathrm{SE})$ from study participants were centrifuged at $4{ }^{\circ} \mathrm{C}$ and serum samples were stored at $-70^{\circ} \mathrm{C}$ until assayed. After exclusions of women who had a serious non-obstetric problem, the cohort of 2379 participants enrolled between 1996 and 2006 were used to select cases and to randomly select the controls.

\subsection{Cases Definition}

Cases of GDM were selected from among participants with a positive glucose challenge test (GCT) at 28 week's gestation $(28.1 \pm 0.1$ weeks, mean $\pm \mathrm{SE})(1 \mathrm{~h}$ plasma glucose concentration $>140 \mathrm{mg} / \mathrm{dl}$ after a $50-\mathrm{g}$ oral glucose load) and with two or more glucose values over the cut points of $95,180,155,140 \mathrm{mg} / \mathrm{dl}$ at fasting, 1,2 , and 3 hours during a $100 \mathrm{~g}$ diagnostic oral glucose tolerance test (OGTT) (the Carpenter/Coustan conversion recommended by ADA) [18]. Women with a positive GCT and fewer than two abnormal glucose values during an OGTT were identified as cases of impaired GCT non-GDM. Gravidae with GDM $(N=80)$, impaired GCT non-GDM $(N=128)$ and 557 normal GCT (con- trols, glucose $\leq 140 \mathrm{mg} / \mathrm{dl}$ at GCT) who were randomly selected from the cohort (SAS procedure, PROC SURVEYSELECT) were included in the study.

\subsection{Analytic Procedures}

Serum adiponectin concentrations were measured by ELISA. The sensitivity for the assay was $0.78 \mathrm{ng} / \mathrm{ml}$ and the antibody pair used was specific to Human Adiponectin and does not significantly cross-react with other cytokine or hormone molecules according to the manufacturer (Human adiponectin ELISA kit, Upstate Chemicon/Millipore, Billerica, MA). The intra and inter adiponectin assay coefficient of variation was $5.8 \%$ and $7.8 \%$ respectively.

\subsection{Statistical Analysis}

Univariate statistics were calculated for continuous variables and Chi-square test was used for categorical variables. Analysis of variance (ANOVA) was used to assess the significance of linear trends among groups with varying degrees of hyperglycemia (cases) to the controls; pairwise contrasts (cases to controls) also were computed. Because of the nature of case-control design, we examined ethnic differences in serum adiponectin concentration by ANOVA comparing mean adiponectin levels of African Americans, Hispanics and Caucasians within each of the case groups and within the control group. We also computed the overall ethnic difference, pooling cases and controls.

Multiple polytomous logistic regression was used to estimate the relation between gestational hyperglycemia (dependant variable coded as GDM, impaired GCT non-GDM vs. normal GCT) and hypoadiponectinemia, defined as serum adiponectin concentration in the lowest tertile (independent variable, coded as lowest tertile vs. tertiles 2 and 3 pooled). This is an extension of traditional logistic regression which models multiple level outcomes so that odds ratios (OR) and adjusted odds ratios (AOR), say for GDM and impaired GCT non-GDM versus controls, are estimated in the same model. Separate models were fit for entry and the $3^{\text {rd }}$ trimester. Odds ratios, adjusted odds ratios, the $\mathrm{p}$ for trend and $95 \%$ confidence intervals (95\% CIs) were computed from the logistic regression coefficients and their corresponding covariance matrices. Polytomous logistic regression also was used to examine differences in adiponectin, with maternal obesity (BMI $<30$ vs. BMI $\geq 30$ ) with and without hyperglycemia. Potential confounding variables including maternal age, ethnicity, parity and cigarette smoking and/or pregravid BMI were controlled in multivariable models accordingly. All statistical analyses were performed using SAS v.9.1 (SAS Institute, Inc., Cary, NC). 


\section{RESULTS}

Maternal characteristics are shown in Table 1. As expected, patients with GDM or impaired GCT non-GDM were older and had higher pregravid BMIs ( $p$ for trend $<0.0001$ for each). There were more nulliparas among controls (41.8\%) compared to GDM (33.8\%) or impaired GCT non-GDM $(30.5 \%, p<0.05)$. Plasma concentrations of glucose, insulin, C-peptide during the $1 \mathrm{hr}$ GCT were increased and exhibited a graded relation between case groups and controls ( $p$ for trend $<0.0001$ for each). Differences in other variables including gestational age at sampling were not statistically significant $(p>0.05$ for each). Ethnic differences at entry differed marginally ( $p$ $=0.066)$.
After adjusted for several confounding variables, serum adiponectin concentrations were significantly different among cases and controls ( $p$ for trend $<0.001$ for each), the lowest level was found in the GDM group, followed by impaired GCT non-GDM, and the controls (vs. GDM $p<0.01$, vs. impaired GCT non-GDM $p<$ 0.05 ) at entry and similar data were obtained at the $3^{\text {rd }}$ trimester (Table 1). Adiponectin levels were significantly decreased by the $3^{\text {rd }}$ trimester compared to entry in each of the three groups ( $p<0.05$ for each, data not shown).

\subsection{Hypoadiponectinemia: Risk of GDM and Impaired GCT Non-GDM}

After multivariable adjustment, at entry to care, $50 \%$

Table 1. Characteristics of study subjects.

\begin{tabular}{|c|c|c|c|c|}
\hline & GDM & Impaired GCT non-GDM & Controls & $P$ for Trend \\
\hline$N$ & 80 & 128 & 557 & \\
\hline Age (yr) & $26.14 \pm 0.54$ & $24.70 \pm 0.42^{a}$ & $22.03 \pm 0.21^{b, c}$ & $<0.0001$ \\
\hline Pre-pregnant BMI (kg/m²) & $30.00 \pm 0.64$ & $26.53 \pm 0.50^{b}$ & $25.49 \pm 0.25^{b}$ & $<0.0001$ \\
\hline Obesity (BMI $\geq 30$ ) & $34(42.5)$ & $32(25.0)$ & $102(18.3)$ & $<0.0001$ \\
\hline Nulliparas & $27(33.8)$ & $39(30.5)$ & $233(41.8)$ & 0.033 \\
\hline Cigarette smoking & $16(20.0)$ & $29(22.7)$ & $97(17.4)$ & 0.365 \\
\hline \multicolumn{5}{|l|}{ Ethnicity } \\
\hline Hispanic & $47(58.9)$ & $60(46.9)$ & $271(48.7)$ & \\
\hline African American & $15(18.8)$ & $48(37.5)$ & $188(33.8)$ & \\
\hline Caucasian & $18(22.5)$ & $20(15.6)$ & $98(17.6)$ & 0.066 \\
\hline \multicolumn{5}{|l|}{ Glucose challenge test $(1 \mathrm{hr})$} \\
\hline Glucose (mmol/l) & $9.36 \pm 0.13$ & $8.44 \pm 0.09^{b}$ & $5.84 \pm 0.05^{b, c}$ & $<0.0001$ \\
\hline Insulin (pmol/1) & $752 \pm 65$ & $863 \pm 48$ & $592 \pm 24^{b, c}$ & $<0.0001$ \\
\hline C-Peptide (nmol/l) & $3.73 \pm 0.24$ & $3.62 \pm 0.18$ & $2.98 \pm 0.09^{d, e}$ & $<0.0001$ \\
\hline \multicolumn{5}{|l|}{ Adiponectin $(\mu \mathrm{g} / \mathrm{ml})$} \\
\hline Entry & $11.49 \pm 0.74$ & $13.18 \pm 0.59$ & $14.67 \pm 0.28^{b, f}$ & $<0.001$ \\
\hline $3^{\text {rd }}$ trimester & $10.17 \pm 0.69$ & $10.73 \pm 0.53$ & $12.45 \pm 0.26^{d, e}$ & 0.05 \\
\hline \multicolumn{5}{|c|}{ Gestational age at blood sampling (wks) } \\
\hline Entry & $16.79 \pm 0.59$ & $16.10 \pm 0.46$ & $17.35 \pm 0.21$ & 0.154 \\
\hline $3^{\text {rd }}$ Trimester & $30.53 \pm 0.37$ & $29.99 \pm 0.29$ & $30.50 \pm 0.14$ & 0.538 \\
\hline Medicaid & $78(97.5)$ & $125(97.7)$ & $546(98.0)$ & 0.932 \\
\hline Gestational age at delivery & $38.33 \pm 0.23$ & $38.61 \pm 0.19$ & $38.54 \pm 0.09$ & 0.592 \\
\hline Infant birth weight $(\mathrm{g})$ & $3379 \pm 46$ & $3251 \pm 36$ & $3209 \pm 18^{a, f}$ & 0.031 \\
\hline
\end{tabular}

Data are mean $\pm \mathrm{SE}$ or $n(\%), P$ for linear contrasts from ANOVA, or Mantel-Haenszel chi-square test. GDM, gestational diabetes mellitus; Impaired GCT non-GDM, glucose $>140 \mathrm{mg} / \mathrm{dl}$ during a glucose challenge test (GCT) at 28 week's gestation and a normal diagnostic oral glucose tolerance test; Normal controls, glucose $\leq 140 \mathrm{mg} / \mathrm{dl}$ during a glucose challenge test (same in Tables 2-4). Infant birth weight was adjusted for gestational age at delivery. ${ }^{a} p<0.05$ vs. GDM; ${ }^{b} p<0.0001$ vs. GDM; ${ }^{c} p<0.0001$ vs. Impaired GCT non-GDM; ${ }^{d} p<0.01$ vs. Impaired GCT non-GDM; ${ }^{p} p<0.01$ vs. GDM; ${ }^{f} p<0.05$ vs. Impaired GCT non-GDM. 
cases with GDM and 38\% of cases with impaired GCT non-GDM compared with $29.6 \%$ of controls were in the lowest tertile of adiponectin, i.e. below $10.7 \mu \mathrm{g} / \mathrm{ml}$ (hypoadiponectinemia). Hypoadiponectinemia was associated with a 2.5 times increased risk of developing GDM; risk was likewise increased by 1.58 times in cases with impaired GCT non-GDM (Table 2) versus controls (model 1. $p$ for trend $<0.0001$ ). Additional adjustment for pregravid BMI (model 2) did not greatly alter effect of adiponectin on hyperglycemia. Similar associations were observed in the $3^{\text {rd }}$ trimester between hypoadiponectinemia and the risk of GDM or impaired GCT non-GDM (Table 2, models 1 and 2).

\subsection{Hypoadiponectinemia, Hyperglycemia and Maternal Obesity}

Adiponectin concentrations were negatively correlated with pregravid BMI $(r=-0.29, p<0.0001$ for entry and $r=$ $-0.24, p<0.001$ for the $3^{\text {rd }}$ trimester). We examined the relationship of hypoadiponectinemia to gestational hyperglycemia with and without maternal obesity (BMI $\geq$ 30 and BMI < 30) (Table 3) using non-obese controls as the reference group. At entry, a reduced adiponectin level was significantly associated with GDM (AOR 2.58, 95\% CI $1.35,4.92$ ) and with impaired GCT non-GDM (AOR $1.61,95 \%$ CI 1.01, 2.61) in non-obese women. Among

Table 2. The association of hypoadiponectinemia with GDM and impaired GCT non-GDM.

\begin{tabular}{|c|c|c|c|}
\hline & GDM & Impaired GCT non-GDM & Controls \\
\hline \multicolumn{4}{|l|}{ Entry Adiponectin tertile $(\mu \mathrm{g} / \mathrm{ml})^{a}$} \\
\hline Lowest (<10.7), $N(\%)$ & $40(50.0)$ & $49(38.3)$ & $165(29.6)$ \\
\hline Middle $(10.7-16.3), N(\%)$ & $23(28.8)$ & $41(32.0)$ & $192(34.5)$ \\
\hline Highest (>16.3), $N(\%)$ & $17(21.3)$ & $38(29.7)$ & $200(35.9)$ \\
\hline Model 1 AOR $(95 \% \mathrm{CI})^{b}$ & $2.68(1.62,4.41)$ & $1.58(1.05,2.38)$ & 1.00 \\
\hline Model 2 AOR $(95 \% \mathrm{CI})^{c}$ & $1.92(1.14,3.24)$ & $1.53(1.02,2.33)$ & 1.00 \\
\hline \multicolumn{4}{|c|}{$3^{\text {rd }}$ Trimester Adiponectin tertile $(\mu \mathrm{g} / \mathrm{ml})^{d}$} \\
\hline Lowest $(<9.0), N(\%)$ & $39(50.7)$ & $53(41.4)$ & $149(28.4)$ \\
\hline Middle $(9.0-13.5), N(\%)$ & $20(25.9)$ & $40(31.6)$ & $185(35.2)$ \\
\hline Highest (>13.5), $N(\%)$ & $18(23.4)$ & $35(27.1)$ & $191(36.4)$ \\
\hline Model 1 AOR $(95 \% \mathrm{CI})^{b}$ & $2.53(1.53,4.19)$ & $1.59(1.06,2.40)$ & 1.00 \\
\hline Model 2 AOR $(95 \% \mathrm{CI})^{c}$ & $2.03(1.21,3.42)$ & $1.58(1.04,2.40)$ & 1.00 \\
\hline
\end{tabular}

AOR, adjusted odds ratio; CI, Confidence interval. ${ }^{a}$ The lowest tertile of serum adiponectin concentration at entry was comparing to other tertiles pooled; ${ }^{b}$ Model 1 , models were adjusted for age, parity, ethnicity and cigarette smoking, $p$ for trend $<0.0001$; ${ }^{c}$ Model 2 , additional adjustment for pre-pregnant BMI, $p$ for trend $<0.01{ }^{d}$ The lowest tertile of serum adiponectin concentration at the $3^{\text {rd }}$ trimester was compared to other tertiles pooled.

Table 3. Decreased serum adiponectin concentrations, hyperglycemia and maternal obesity ${ }^{a, b}$.

\begin{tabular}{|c|c|c|c|c|}
\hline \multirow[b]{3}{*}{ Non-obese (BMI < 30) } & \multicolumn{4}{|c|}{ Adiponectin $^{c}$} \\
\hline & \multicolumn{2}{|c|}{ Entry $<10.7 \mu \mathrm{g} / \mathrm{ml}$} & \multicolumn{2}{|c|}{ Week $28<9.0 \mu \mathrm{g} / \mathrm{ml}$} \\
\hline & $\mathrm{n}(\%)$ & $\operatorname{AOR}(95 \% \mathrm{CI})$ & $n(\%)$ & $\operatorname{AOR}(95 \% \mathrm{CI})$ \\
\hline Controls & $115(25.2)$ & reference & $113(26.4)$ & reference \\
\hline Impaired GCT non-GDM & $32(33.3)$ & $1.61(1.01,2.61)$ & $40(38.8)$ & $1.60(1.01,2.56)$ \\
\hline GDM & $20(43.5)$ & $2.58(1.35,4.92)$ & $19(41.3)$ & $2.11(1.11,4.06)$ \\
\hline \multicolumn{5}{|l|}{ Obese (BMI $\geq 30$ ) } \\
\hline Controls & $52(51.0)$ & $3.20(2.04,5.03)$ & $40(41.2)$ & $1.73(1.08,2.76)$ \\
\hline Impaired GCT non-GDM & $19(59.4)$ & $4.81(2.25,10.28)$ & $16(53.3)$ & $2.67(1.24,6.75)$ \\
\hline GDM & $20(58.8)$ & $4.90(2.33,10.30)$ & $20(64.5)$ & $4.55(2.08,9.95)$ \\
\hline
\end{tabular}

${ }^{a}$ Models were adjusted for age, parity, ethnicity and cigarette smoking; ${ }^{b} p$ for trend $<0.0001$ for all models; ${ }^{c}$ The lowest tertile of adiponectin concentration at entry $(<10.7 \mu \mathrm{g} / \mathrm{ml})$ and week $28(<9.0 \mu \mathrm{g} / \mathrm{ml})$. 
the obese, hypoadiponectinemia was associated with nearly 5-fold increased risks of GDM (AOR 4.90, 95\% CI $2.33,10.30)$ and impaired GCT non-GDM (AOR 4.81, $95 \%$ CI $2.25,10.28)$. There was a 3 fold increased risk in obese women without hyperglycemia (AOR 3.20, 95\% CI 2.04, 5.03). Although adjusted odds ratios were lower in non-obese than in obese women (Table 3), they were not statistically different in that $95 \%$ confidence intervals all overlapped. During $3^{\text {rd }}$ trimester, hypoadiponectinemia was also associated with GDM or impaired GCT non-GDM regardless of whether or not the woman was obese.

\subsection{Ethnic Differences in Serum Adiponectin Concentration}

During the $3^{\text {rd }}$ trimester, concentrations of adiponectin were consistently and significantly lower in African American cases with GDM, as well as in cases with impaired GCT non-GDM when compared to Hispanics or Caucasians of the same case group; this also held true for the controls (model was fully adjusted for confounding variables) ( $p<0.05$ or $p<0.01$ for each, Table 4). At entry the only significant difference observed was for African American with impaired GCT non-GDM (10.82 $\pm 0.89)$ in comparison to their Hispanic counterparts $(15.04 \pm 0.80, p<0.01)$.

We also compared the changes in serum adiponectin level between entry and the $3^{\text {rd }}$ trimester by ethnicity, pooling cases and controls. During the $3^{\text {rd }}$ trimester, adiponectin concentration decreased significantly $(p<0.05)$ within each ethnic group, African Americans (-3.13 \pm $0.49)$, Hispanics $(-2.27 \pm 0.39)$ and Caucasians $(-1.43 \pm$ $0.66)$.

\section{DISCUSSION}

In this case-control study nested within a large prospective cohort in a well characterized multiethnic group of subjects, we were able to demonstrate a unique graded association between hypoadiponectinemia during early gestation and risk for overt GDM and for less severe hyperglycemia, e.g., impaired GCT non-GDM. This relationship persisted during the $3^{\text {rd }}$ trimester.

Decreased serum adiponectin in early pregnancy predicts an increased risk to develop GDM [6]; cross sectional studies from later pregnancy also have found lower levels with GDM [7-9]. Our data are in agreement with these findings. Entry hypoadiponectinemia is associated with a more than 2-fold increased risk of GDM and this relation persists into the $3^{\text {rd }}$ trimester, after multivariable adjustment (Table 2).

Apart from GDM, little is known about whether or not low serum adiponectin increases risk of developing mild to moderate hyperglycemia. Maternal adiponectin correlates negatively with plasma glucose (fasting and during OGTT) in women with mild hyperglycemia without overt GDM [19]. However, the measurements were taken late in gestation ( 28 weeks) and did not include controls with normal glucose tolerance [19]. In contrast, another study of adiponectin in late gestation found no difference between controls $(15.54 \mu \mathrm{g} / \mathrm{ml})$ and women with impaired glucose tolerance $(15.06 \mu \mathrm{g} / \mathrm{ml})$ [9]. We found hypoadiponectinemia at entry to be significantly associated with a 1 .6-fold increased risk of impaired GCT non-GDM and the results were not greatly altered after adjustment for maternal BMI (Table 2). These effects persisted, despite the fact that the concentration of adiponectin decreased during the $3^{\text {rd }}$ trimester in the both cases and controls (Tables 1 and 2). The strength of this

Table 4. Ethnic differences in maternal serum adiponectin concentration by glycemic group.

\begin{tabular}{cccc}
\hline Adiponectin $(\mu \mathrm{g} / \mathrm{ml})$ & African American & Hispanic & Caucasian \\
\hline GDM $(\boldsymbol{N})$ & $(15)$ & $(47)$ & $11.32 \pm 0.86$ \\
Entry & $11.23 \pm 1.56$ & $10.00 \pm 0.71$ & $12.13 \pm 1.41$ \\
$3^{\text {rd }}$ trimester & $8.26 \pm 1.36^{a}$ & $(60)$ & $(20)$ \\
Impaired GCT non-GDM $(\boldsymbol{N})$ & $(48)$ & $15.04 \pm 0.80$ & $13.37 \pm 1.39$ \\
Entry & $10.82 \pm 0.89^{b}$ & $11.98 \pm 0.74$ & $11.28 \pm 1.26$ \\
$3^{\text {rd }}$ trimester & $8.95 \pm 0.84^{b}$ & $(271)$ & $(98)$ \\
Controls $(\boldsymbol{N})$ & $(188)$ & $14.74 \pm 0.39$ & $15.00 \pm 0.66$ \\
Entry & $14.41 \pm 0.47$ & $12.66 \pm 0.37$ & $14.08 \pm 0.64$
\end{tabular}

Models were adjusted for maternal age, parity, cigarette smoking and pre-pregnant BMI. ${ }^{a} p<0.05$ vs. Caucasian; ${ }^{b} p<0.01$ vs. Hispanic; ${ }^{c} p<0.01$ vs. Caucasian; ${ }^{d} p<0.05$ vs. Hispanic. 
study is that we are able to show graded associations between varying degree of hyperglycemia and adiponectin which extend previous findings. Our results suggest that decreased adiponectin during early pregnancy is a useful predictor/marker for both GDM and less severe hyperglycemia non-GDM.

Adiponectin is inversely associated with body composition and obesity in non-pregnant subjects [20,21]. Excess fat mass or visceral adipose tissue exerts a negative feedback on the expression and production of adiponectin $[2,4,16]$. Data on whether the increased risk of GDM with low adiponectin is independent of maternal obesity are inconsistent $[6,7,9]$. We observed that the adjusted odds ratio was increased even in obese women with a normal GCT and the association between low adiponectin and hyperglycemia did not change significantly regardless of whether women were obese or not (Table 3).

The prevalence of obesity and metabolic syndrome is greater in non-pregnant African American women than Caucasians $[13,14]$. The ethnic differences in insulin secretion and resistance are detectable during childhood and adolescence [22]. Environmental factors contribute to some but not all ethnic disparities [13,14]. Adiponectin has been implicated as an anti-inflammatory and antiantherogenic hormone but data on ethnic differences in adiponectin during pregnancy are limited. Khoo et al. reported that adiponectin concentration was different among non-pregnant women in several Asian ethnic groups [23]. Our data showed that African American women had significantly lower adiponectin concentrations than Caucasians and/or Hispanics (Table 4). This difference is consistent among each of three glycemic groups during $3^{\text {rd }}$ trimester, but at entry to care the difference was only detectable in the group with impaired GCT non-GDM. To our knowledge, the current study is the first demonstration of ethnic differences in serum adiponectin for pregnant women in USA.

Why pregnant African American women have lower adiponectin is unclear. Adiponectin is down regulated by multiple factors, including insulin resistance and the inflammation which characterizes late pregnancy $[2,15]$. Previously we found non-diabetic African American women to have higher insulin levels and increased insulin resistance but reduced insulin clearance [24]. Thus, a possible explanation for the ethnic difference during the $3^{\text {rd }}$ trimester could be a response to a different degree of insulin resistance in African American where the greater the insulin resistance, the greater the suppression of adiponectin production.

In summary, hypoadiponectinemia during early and late pregnancy was strongly associated with risk of various degrees of gestational hyperglycemia, including GDM and less severe hyperglycemia non-GDM. This association did not change significantly in women with or without obesity. There is a detectable ethnic difference in adiponectin concentration during late gestation, in that African American women regardless of whether they are hyperglycemic or normoglycemic have lower adiponectin levels than Caucasian or Hispanic women. These data suggest that hypoadiponectinemia may play a significant pathophysiological role in the development of GDM and of less severe gestational hyperglycemia and may have clinical relevance with respect to GDM and less severe hyperglycemia risk prediction.

\section{ACKNOWLEDGEMENTS}

This work was supported by grants from the National Institute of Child Health and Human Development (HD18269 and HD38329, T.O.S.) and National Institute of Diabetes and Digestive and Kidney Diseases (1R21DK078865-01 and 5R21DK078865-02, X.C.). We thank the staff of the Osborn Family Health Center, Our Lady of Lourdes Hospital for providing access to patients, Deborah Cruz for typing the manuscript.

\section{REFERENCES}

[1] Okui, H., Hamasaki, S., Ishida, S., Kataoka, T., Orihara, K., Fukudome, T., et al. (2008) Adiponectin is a better predictor of endothelial function of the coronary artery than HOMA-R, body mass index, immunoreative insulin, or triglycerides. International Journal of Cardiology, 26, 53-61. doi:10.1016/j.ijcard.2007.03.116

[2] Retnakaran, R., Hanley, A.J.G., Raif, N., Hirning, C.R., Connelly, P.W., Sermer, M., et al. (2005) Adiponectin and beta cell dysfunction in gestational diabetes: Pathophysiological implications. Diabetologia, 48, 993-1001. doi:10.1007/s00125-005-1710-x

[3] Krakoff, J., Funahashi, T., Stehouwer, C.D.A., Schalkwijk, C.G., Tanaka, S., Matsuzawa, Y., et al. (2003) Inflammatory markers, adiponectin, and risk of type 2 diabetes in the Pima Indian. Diabetes Care, 26, 1745-1751. doi:10.2337/diacare.26.6.1745

[4] Tan, K.C.B., Xu, A., Chow, W.S., Lam, M.C.W., Ai, V.H.G., Tam, S.C.F., et al. (2004) Hypoadiponectinemia is associated with impaired endothelium-dependent vasodilation. The Journal of Clinical Endocrinology and Metabolism, 89, 765-769. doi:10.1210/jc.2003-031012

[5] Duncan, B.B., Schmidt, M.I., Pankow, J.S., Bang, H., Couper, D., Ballantyne, C.M., et al. (2004) Adiponectin and the development of type 2 diabetes: The atherosclerosis risk in communities study. Diabetes, 53, 2473-2478. doi:10.2337/diabetes.53.9.2473

[6] Williams, M.A., Qiu, C., Muy-Rivera, M., Vadachkoria, S., Song, T. and Luthy, D.A. (2004) Plasma adiponectin concentrations in early pregnancy and subsequent risk of gestational diabetes mellitus. The Journal of Clinical Endocrinology and Metabolism, 89, 2306-2311. doi:10.1210/jc.2003-031201

[7] Ranheim, T., Haugen, F., Staff, A.C., Braekke, K., Harsem, 
N.K. and Drevon, C.A. (2004) Adiponectin is reduced in gestational diabetes mellitus in normal weight women. Acta Obstetricia et Gynecologica Scandinavica, 83, 341347.

[8] Tsai, P.J., Yu, C.H., Hsu, S.P., Lee, Y.H., Huang, I.T., et al. (2005) Maternal plasma adiponectin concentrations at 24 to 31 weeks of gestation: Negative association with gestational diabetes mellitus. Nutrition, 21, 1095-1099. doi:10.1016/j.nut.2005.03.008

[9] Retnakaran, R., Hanley, A.J.G., Raif, N., Connelly, P.W., Sermer, M. and Zinman, B. (2004) Reduced adiponectin concentration in women with gestational diabetes: A potential factor in progression to type 2 diabetes. Diabetes Care, 27, 799-800. doi:10.2337/diacare.27.3.799

[10] The HAPO Study Cooperative Research Group. (2008) Hyperglycemia and adverse pregnancy outcomes. The New England Journal of Medicine, 358, 1991-2002. doi:10.1056/NEJMoa0707943

[11] Catalano, P.M., Hoegh, M., Minium, J., Huston-Presley, L., Bernard, S., et al. (2006) Adiponectin in human pregnancy: Implications for regulation of glucose and lipid metabolism. Diabetologia, 49, 1677-1685. doi:10.1007/s00125-006-0264-x

[12] Chen, X., Scholl, T.O., Leskiw, M., Savaille, J. and Stein, T.P. (2010) Differences in maternal circulating fatty acid composition and dietary fat intake in women with gestational diabetes mellitus or mild gestational hyperglycemia. Diabetes Care, 33, 2049-2054. doi:10.2337/dc10-0693

[13] Ervin, R.B. (2009) Prevalence of metabolic syndrome among adults 20 years of age and over, by sex, age, race and ethnicity, and body mass index: United States, 2003-2006. National Health Statistics Report, 5, 1-7.

[14] Cossrow, N. and Falkner, B. (2004) Race/ethnic issues in obesity and obesity-related comorbidities. The Journal of Clinical Endocrinology and Metabolism, 89, 2590-2594. doi:10.1210/jc.2004-0339

[15] Karastergiou, K., Mohamed-Ali, V., Jahangiri, M. and Kaski, J.C. (2009) Adiponectin for prediction of cardiovascular risk? The British Journal of Diabetes and Vascular Disease, 9, 150-154. doi:10.1177/1474651409341326
[16] Mather, K.J., Funahashi, T., Matsuzawa, Y., Edelstein, S., Bray, G.A., et al. (2008) Adiponectin, change in adiponectin, and progression to diabetes in the diabetes prevention program. Diabetes, 57, 980-986. doi:10.2337/db07-1419

[17] Webster, B.H. and Bishaw, A. (2006) Income, earnings, and poverty data from the 2005 American Community Survey. http:www.census.gov/prod/2006pubs/acs-02.pdf

[18] American Diabetes Association. (2000) Clinical practice recommendations 2000: Position statement. Gestational diabetes mellitus. Diabetes Care, 23, S77-S79.

[19] Lowe, L.P., Metzger, B.E., Lowe, W.L. Jr., Dyer, A.R., McDade, T.W. and McIntyre, H.D. (2010) Inflammatory mediators and glucose in pregnancy: Results from a subset of the hyperglycemia and adverse pregnancy outcome (HAPO) study. Journal of Clinical Endocrinology and Metabolism, 95, 5427-5434. doi:10.1210/jc.2010-1662

[20] Kanaya, A.M., Harris, T., Goodpaster, B.H., Tylavsky, F. and Cummings, S.R. (2004) Adipocytokines attenuate the association between visceral adiposity and diabetes in older adults. Diabetes Care, 27, 1375-1380. doi:10.2337/diacare.27.6.1375

[21] Winzer, C., Wagner, O., Festa, A., Schneider, B., Roden, M., et al. (2004) Plasma adiponectin, insulin sensitivity, and subclinical inflammation in women with prior gestational diabetes mellitus. Diabetes Care, 27, 1721-1727. doi:10.2337/diacare.27.7.1721

[22] Yanovski, J.A., Yanovski, S.Z., Filmer, K.M., Hubbard, V.S., Avila, N., et al. (1996) Differences in body composition of black and white girls. The American Journal of Clinical Nutrition, 64, 833-839.

[23] Khoo, C.M., Sairazi, S., Taslim, S., Gardner, D., Wu, Y., et al. (2011) Ethnicity modifies the relationships of insulin resistance, inflammation, and adiponectin with obesity in a multiethnic Asian population. Diabetes Care, 34, 1120-1126. doi:10.2337/dc10-2097

[24] Chen, X. and Scholl, T.O. (2002) Ethnic differences in C-peptide/insulin/glucose dynamics in young pregnant women. The Journal of Clinical Endocrinology and Metabolism, 87, 4642-4646. doi:10.1210/jc.2001-011949 